various psychiatric disorders, due to the virus brain tropism and associated psychosocial factors. However, there are still limited data regarding the psychiatric needs and mental health care of these patients. The present study seeks to evaluate the changes in liaison psychiatry, patients' characteristics and outcomes, brought on by SARS-CoV-2.

Method. We retrospectively analyzed liaison psychiatry patients' characteristics during the second wave of COVID-19 pandemic, between October and December 2020, in comparison with the same trimester pre-COVID-19 in 2019.

Result. At admission to hospital, we found no differences between liaison psychiatry pre-COVID-19 $(\mathrm{n}=96)$ and COVID-19 period $(n=114)$ groups, for age $(p=0.35)$, gender $(p=0.96)$, or hospital resource utilization in the previous year : number of hospital admissions $(\mathrm{p}=.40)$, Accident and Emergency presentations ( $\mathrm{p}$ $=0.61)$, outpatients clinics $(\mathrm{p}=0.20)$. There was, however, a significant association between pre-COVID-19 vs COVID-19 period groups and psychiatric diagnosis during hospitalization $(\mathrm{X} 2(5)=$ 11.56, $\mathrm{p}=0.04)$, influencing overall respective rates of delirium/ dementias (29.2 vs $42.1 \%$ ), mood disorders (18.8 vs $26.3 \%$ ), anxiety and stress related disorders (22.9 vs $14.0 \%)$, psychoactive substance use disorders (12.5 Vs 9.6 \%) and psychotic disorders (10.4 vs $2.6 \%$ ). Mortality rate in pre-COVID-19 Vs COVID-19 period increased from $5.2 \%$ to $15.8 \%(\mathrm{X} 2(1)=5.98, \mathrm{p}=0.01)$, mostly due to increased mortality in COVID-19 patients (7.3 vs $30.3 \%, \mathrm{p}<0.001)$. Referrals to liaison psychiatry increased by $18.8 \%$ during the COVID-19 period, $28.8 \%$ of referred patients were positive for SARS-CoV-2 and time to referral was positively correlated with hospital length of stay $(\rho=0.60, p<0.001)$. In the COVID-19 period, fewer patients were referred to outpatient psychiatric follow-up after discharge from hospital $(p<0.001)$, and delayed discharges for social reasons were of shorter duration $(\mathrm{p}=0.001)$.

Conclusion. Our findings are consistent with emerging data on comorbid or superimposed mental illness in hospitalized patients with COVID-19, showing a strong impact in neuropsychiatric mani-festations, clinical prognosis and mortality, as well as clinical management. Consideration should also be given to adequate staffing of psychiatry liaison services during the pandemic, in order to attend and safely manage general hospital inpatients mental health needs.

\section{Physical health of patients under EIP - a service evaluation}

Anu Priya ${ }^{1 \star}$, Keerthan Dhanasekar ${ }^{2}$, James Hill-Cousins ${ }^{2}$, Mudasar Aziz ${ }^{2}$, Osama Suleiman ${ }^{2}$, Katherine Mills ${ }^{2}$ and Megan Lloyd ${ }^{2}$

${ }^{1}$ Bassetlaw Mental Health Services and ${ }^{2}$ Sheffield University ${ }^{*}$ Corresponding author.

doi: 10.1192/bjo.2021.897

Aims. The aim of the project was to get a baseline of the number of patients who have had blood tests, ECG and physical health observations completed in the past 12 months.

Method. There are 30 patients under Early Intervention in Psychosis team at Bassetlaw Hospital , Nottinghamshire. The elctronic notes and blood reporting system were checked for each of the patients, to get the data on blood test results , ECG reports and Physical health (Blood pressure, heart rate and weight) .

Result. It was noted that $19 / 30$ patients had Blood tests completed, 14/30 had ECG completed and 19/30 had physical health checks completed. All these patients except one were on antipsychotic medications.
Conclusion. Further work is still required in getting $100 \%$ results for all these different variables. This may include the need to review the process of how we engage the patients for physical healthcare checks. With the inclusion of a physical healthcare worker now, we might be able to improve results. Hence this evaluation would be redone in a years' time.

\section{Balint style case-based discussion group for medical students in Bassetlaw Hospital}

Anu Priya* and Hardev Bhogal

Bassetlaw Mental Health Services

${ }^{\star}$ Corresponding author.

doi: 10.1192/bjo.2021.898

Aims. To evaluate feedback from Balint style case based discussion groups and to reflect on learning points.

We have three medical students from Sheffield University on six week psychiatry placements at Bassetlaw Hospital and we get eighteen students in a year. In order to further develop their approach towards reflection and their understanding towards the doctor patient relationship we developed the Balint style case based discussion group, and each group of students attend three sessions during their placement.

Method. The groups are held on a weekly basis and consist of the three medical students and 1-2 facilitators. As the group is small one of the facilitators may participate with the students for the Balint process and to help encourage the students. Following completion of the third session of the discussion group we gain written feedback from the students. A total of 17 feedbacks have been reviewed over the period of November 2018 -November 2019.

Result. 16 students stated that this was their first experience at Balint Group and all except one student felt that they were given a good introduction about Balint groups before starting. When asked about the most significant thing that they have learnt in the group, the majority of students marked reflecting feelings to improve relationships with patients, exploring why they feel a certain way with patients and that the doctor patient relationship can affect the consultation.

One student stated that they would not recommend it to colleagues as they felt it was relevant more to doctors rather than medical students. Another student recommended having more people in a group.

Conclusion. Overall, it has been a positive experience with the medical students during the groups and with feedbacks. We have reflected on difficult topics like bereavement, fantasized about the purpose of a patient's delusion and shared the joy of a patient who was discharged after a long stay. While we think we have been able to teach the students some tips on reflection, we ourselves have been able to reflect on certain topics we would not have if not raised by the students. Some medical students have contacted the larger Balint Group in Sheffield for further sessions. Considering our experience, we will continue with the sessions at Bassetlaw Hospital.

\section{Adult ADHD clinics in north Wales - case load prevalance \& compliance with nice guidelines (quality evaluation project) \\ Jawad Raja ${ }^{1 \star}$, Zeenish Azhar ${ }^{2}$ and Alberto Salmoiraghi ${ }^{3}$ \\ ${ }^{1}$ ST6 GA Trainee Betsi Cadwaladr University Health Board; ${ }^{2}$ CT3 \\ Trainee Betsi Cadwaladr University Health Board and ${ }^{3}$ Medical \\ Director Betsi Cadwaladr University Health Board \\ ${ }^{\star}$ Corresponding author.}

doi: 10.1192/bjo.2021.899 
Aims. Measure compliance with National Institute for Health and Care Excellence (NICE) recommendations in four Adult CMHT's Guide further service development.

We audited the case notes of 20 patients each currently under care of 9 General Adult Consultants across 6 CMHT's in East side of North Wales against NICE standards using an adapted version of the ADHD audit support tool.

My role in the Project \& How does this represent my practice? I was the audit and overall lead for this project

I formulated the audit tool and registered my project with Audit Registration Team.

I lead data collection and compilation of results.

Method. Overall, this is the first audit of Adult ADHD Services in East side of North Wales.

It established good compliance with NICE guidance for assessment and treatment.

NICE has expressed the need for full mental health and social assessment including full history and physical examination prior to the drug treatment.

Good compliance was observed in using \& documenting Diagnostic Criteria (DSM-IV and/or ICD-10).

There were deficiencies in conducting or arranging recommended physical examination \& side effect monitoring.

Drug treatment was the first line of treatment in the majority of cases.

Antipsychotics were used in some patients referred for ADHD assessment, despite the fact that NICE has ruled out the use of antipsychotic drugs in treatment of core symptoms of ADHD.

Result. Prevalence of Adult ADHD clinician case load in Wrexham and Fintshire Counties.

Diagnosis of Adult ADHD according to ICD 10 \& DSM IV Guidelines.

Pre treatment screening of physical health for ADHD patients.

Side effects monitoring of patients on stimulant medications.

Conclusion. The finding highlights the need for more effort in educating clinicians about safety and effectiveness of antipsychotics in ADHD.

Comprehensive treatment programmes that address psychological, behavioural, educational and occupational needs should be established.

Development of local ADHD Clinics, support groups and in partnership with the voluntary sector should be encouraged.

It is important that mental health professionals receive appropriate training in assessment, management \& monitoring of ADHD patients with co morbid substance use disorder and other mental illnesses.

BETSI Health Board to participate in national Prescribing Observatory for Mental Health (POMH-UK) Quality Improvement Programme (QIP) focusing on prescribing for ADHD in children, adolescents \& adults.

\section{Clozapine clinics in north Wales - service evaluation audit}

Jawad Raja ${ }^{1 \star}$, Zeenish Azhar ${ }^{2}$ and Masood Malik ${ }^{3}$

${ }^{1}$ ST5 Betsi Cadwaladr University Health Board; ${ }^{2}$ CT2 Betsi Cadwaladr University Health Board and ${ }^{3}$ Clinical Director Betsi Cadwaladr University Health Board

${ }^{\star}$ Corresponding author.

doi: 10.1192/bjo.2021.900

\section{Aims.}

1. Quality of clozapine clinic appointment

2. Effectiveness of clozapine clinic servive
3. Compliance with BCUHB guidelines for physical health monitoring in clozapine clinics

4. We retrospectively audited 40 case notes 10 each from 4 differtent CMHT clozapine clinics

My role in the Project \& How does this represent my practice?

1. I was the audit and overall lead for this project

2. I formulated the audit tool and registered my project with Audit Registration Team

3. I lead data collection and compilation of results

\section{Background.}

1. This audit followed up from a Coroner's investigation for a clozapine clinic patient

2. Clozapine is used for Treatment Resistant Schizophrenia but needs close monitoring due to potentially fatal side effects

3. NICE recommends annual monitoring of weight, blood pressure, waist measurement, blood glucose and plasma lipid levels

\section{Method.}

1. Has the patient been seen in the past year by clinician to monitor response to clozapine treatment?

2. Has the clozapine plasma level been measured during the last year of treatment?

3. Is brief MSE \& Risk assessment documented during review?

4. Has Life style modification advice been provided?

5. Has annual physical health been completed?

6. Has Annual CTP/CPA been completed and documented?

7. Has the patient been allocated a named care coordinator?

8. Has clozapine side effects monitoring been documented?

\section{Conclusion.}

1. Clozapine is a superior medication for the treatment of refractory schizophrenia and is also be effective for other conditions

2. Clozapine is underused due to a variety of barriers related to the drug and its properties, the health care system \& regulatory requirements

3. This service evaluation/quality improvement project provides the framework for clozapine clinics evaluation and recommends strategies for improvement

Service evaluation of primary care mental health support services in north Wales

Jawad Raja ${ }^{1 \star}$, Alberto Salmoiraghi ${ }^{2}$ and Zeenish Azhar $^{3}$

${ }^{1}$ ST5 Betsi Cadwaladr University Health Board; ${ }^{2}$ Medical Director Betsi Cadwaladr University Health Board and ${ }^{3}$ CT3 Trainee Betsi Cadwaladr University Health Board

${ }^{\star}$ Corresponding author.

doi: 10.1192/bjo.2021.901

Aims. Bringing specialist psychiatrist into PCMHT Undertaking initial assessments for people Referred by G.P's Working According to the principle of "Prescribing Interventions"

Decrease number of assessments carried out within secondary Care

Method. County of Wrexham is situated between the lower Dee Valley and the Welsh mountains. It is the largest town in North Wales $(140,000)$

Since 2013, the total new patient referrals to be seen by Wrexham county consultant psychiatrists has consistently risen 